\title{
Advances in Neutrophil Biology:
}

\author{
Clinical Implications
}

Andrew S. Cowburn, MSc, PhD, Alison M. Condliffe, PhD, FRCP, Neda Farahi, PhD, Charlotte Summers, BSc, BM, MRCP, and Edwin R. Chilvers, PhD, FMedSci

Respiratory Medicine Division, Department of Medicine, University of Cambridge School of

Clinical Medicine, Addenbrooke's and Papworth Hospitals, Cambridge, UK

\section{Abstract}

Many lung diseases are characterized by neutrophil-dominated inflammation; therefore, an understanding of neutrophil function is of considerable importance to respiratory physicians. This review will focus on recent advances in our understanding of how neutrophils are produced, how these cells leave the circulation, the molecular events regulating neutrophil activation and, ultimately, how these cells die and are removed. The neutrophil is now recognized as a highly versatile and sophisticated cell with significant synthetic capacity and an important role in linking the innate and adaptive arms of the immune response. One of the key challenges in conditions such as COPD, bronchiectasis, cystic fibrosis, and certain forms of asthma is how to manipulate neutrophil function in a way that does not compromise antibacterial and antifungal capacity. The possession by neutrophils of a unique repertoire of surface receptors and signaling proteins may make such targeted therapy possible.

\section{Keywords}

COPD; inflammatory lung disease; neutrophils; nicotinamide adenine dinucleotide phosphate oxidase

\section{Role of the Neutrophil in Respiratory Disease}

Many airway diseases including COPD, bronchiectasis, bronchiolitis, and cystic fibrosis are characterized by neutrophil infiltration of the airway wall. Likewise, neutrophils are believed to play a fundamental role in acute lung injury (ALI)/ARDS and many of the vasculitides. Considerable observational and experimental data support an association between neutrophils and the severity and progression of the above airways diseases; for example, in COPD neutrophils have been shown to be the most abundant inflammatory cell present in the bronchial wall, bronchial lumen, lung parenchyma, and submucosal glands, 1 and the intensity of the airway and blood neutrophilia, in smokers and ex-smokers, correlates with the rate of decline in $\mathrm{FEV}_{1} .2$ In addition, neutrophils have been demonstrated in bronchial biopsy samples obtained taken during the allergen-induced late asthmatic response, 3 and a strong association has now been established between airway neutrophilia and severe asthma, corticosteroid-resistant asthma, asthma exacerbations, acute fatal asthma, and certain forms of occupational asthma (see Macdowell and Peters4). Where measured, neutrophil numbers in asthma correlate with markers of neutrophil degranulation, implying

\footnotetext{
(C) 2008 American College of Chest Physicians

Correspondence to: Edwin R. Chilvers, PhD, FMedSci, Respiratory Medicine Division, Department of Medicine, University of Cambridge School of Clinical Medicine, Box 157, Addenbrooke's Hospital, Hills Rd, Cambridge, CB2 2QQ, UK; erc24@ cam.ac.uk. The authors have no conflicts of interest relevant to this article to disclose.
} 
that these cells are also activated. While in severe asthma, eosinophils and neutrophils are often found together, neutrophils may gradually replace eosinophils in proportion to the severity and/or duration of the disease, perhaps reflecting the ability of corticosteroids to induce eosinophil apoptosis while inhibiting this process in neutrophils.5 In addition to the direct effects of proteases on airway epithelia, neutrophil elastase has been shown to stimulate transforming growth factor- $\beta$ release from airway smooth-muscle cells, 6 suggesting a potential role in airway remodeling.

While the study of human neutrophils has been hindered by the fact that they are terminally differentiated and short-lived cells not amenable to in vitro genetic manipulation, animal models have provided novel insights into neutrophil biology. A good example of this is the zebra fish model established by Renshaw et al,7 which shares many features of the human innate immune system. Genetic manipulation in this model allows selective expression of green fluorescent protein in zebra fish neutrophils, allowing remarkable real-time imaging of neutrophilic inflammation following localized injury. This system can be manipulated further both genetically and pharmacologically, allowing the identification of novel targets that modulate neutrophil recruitment, function, and physiologic fate in vivo. This article seeks to present some of the most important recent insights into neutrophil biology and show how such information may impact on our understanding of lung disease and future therapies.

\section{Neutrophil Production and Physiologic Fate}

Given the average peripheral blood neutrophil count of $2.5-7.5 \times 10^{9} / \mathrm{L}$ and a circulating half-life of just 6 to $8 \mathrm{~h}$, the bone marrow is required to produce and release $>5-10 \times 10^{10}$ neutrophils every day, with the capacity to increase production still further if needed. Granulocytes and macrophages differentiate from a common committed progenitor cell. Transcriptional profiling studies 8 have suggested that macrophages represent the default myeloid cell and that granulocytes arise through the selective expression of a subset of transcription factors (Egr1, HoxB7, STAT3), granulocyte proteins (eg, S100A8, S100A9, neutrophil elastase), and receptors ( $\mathrm{N}$-formylmethionyl-leucyl-phenylalanine, granulocyte macrophage colony-stimulating factor); this may explain, in part, the surprisingly complex transcriptome in these cells.

Despite their short circulating half-life, human neutrophils, unlike platelets and erythrocytes, appear to be removed from the circulation in a random rather than an age-related manner. However, one mechanism shown to control the release and reuptake of neutrophils in the mouse bone marrow is the interaction of stromal cell-derived factor-1a and its cognate receptor on the neutrophil, CXCR4. Expression of CXCR4 increases as neutrophils age and is thought to result in cells homing to murine bone marrow9; it is unknown whether a similar mechanism promotes removal of aged neutrophils by the human bone marrow.

\section{Neutrophil Migration}

The ability of the neutrophil to "sense" phagocytic bait and migrate along ultrafine chemotactic gradients is astonishing [http://www.flicklife.com/thumb/1_4305.jpg]. The principal signals for neutrophil influx in COPD airways, as judged from sputum and blood analysis, appear to be leukotriene- $\mathrm{B}_{4}$ (acting via the high-affinity leukotriene- $\beta_{4}$ receptor) and interleukin (IL)-8 (acting via the chemokine receptors CXCR1 and CXCR2),10 but other chemotactic factors such as C5a, CXCL1 (growth-related oncogene-a), CXCL5 (epithelial cell neutrophil-activating protein-78), and elastase- $a_{1}$-antitrypsin complexes may also contribute. Classically, IL-8 has been implicated in mediating the influx of neutrophils into the lung in ARDS, 11 but elevated IL- 8 in sputum has also been detected in adults suffering acute asthma exacerbations, where it correlates with the presence of a predominantly neutrophilic inflammatory response. 12 The detection of chemotactic stimuli 
leads to the activation of signaling pathways, cytoskeletal rearrangement, and changes in cell surface molecules that coordinate to facilitate neutrophil migration.

In the lung parenchyma, neutrophils exit the pulmonary circulation at the capillary level, rather than via postcapillary venules, as in the systemic circulation.13 This unique site for neutrophil migration may be amenable to selective interventions. Rheologic considerations including the size, shape, and deformability of neutrophils (a function of cytoskeletal changes) are important determinants of neutrophil transit across the pulmonary capillary network.14 Recruitment of neutrophils from the bronchial circulation may follow the "rolling-tethering" paradigm, reviewed extensively elsewhere15; in this process, the interaction of neutrophil P-selectin glycoprotein ligand-1, E-selectin ligand-1, and Lselectin, and subsequently the $\beta_{2}$-integrins (lymphocyte function-associated antigen- 1 and Mac-1) with their cognate endothelial partners, leads to rolling and subsequently firm cell adhesion/arrest. The involvement of different adhesion molecules also varies according the precise stimulus.16 Once arrested in the circulation, migrating neutrophils must cross the endothelial cell layer and traverse the pericyte sheath and the basement membrane. A recent study 17 has demonstrated that, remarkably, up to $70 \%$ of transmigrating neutrophils cross the endothelial barrier by an active process of engulfment by endothelial cells that is dependent on the small guanosine triphosphatase RhoG. Less is known about the mechanisms of migration through the pericyte sheath and basement membrane, but the existence of regions of reduced matrix protein density within the endothelial cell basement membrane has been reported. These low-density regions were shown to align with the gaps between pericytes and offered preferential sites for neutrophil transmigration.18

Directional leukocyte recruitment (chemotaxis) was shown to be dependent on phosphoinositide 3-kinase (PI3K)- $\gamma$, which belongs to a family of class I PI3Ks.19 Given that the expression of PI3K- $\gamma$ (and PI3K- $\delta$ ) is largely restricted to leukocytes, this has triggered much interest in the therapeutic potential of the newly developed PI3K- $\gamma$ isoformselective inhibitors. Initial studies in mice however, suggest that PI3K- $\gamma$ gene deletion, while effective in suppressing inflammation in a model of rheumatoid arthritis, 20 may impair macrophage recruitment to the lung and defense against Streptococcus pneumoniae. 21 Many chemoattractant molecules such as formylated bacterial peptides have promiscuous effects on neutrophil function, inducing predominantly chemotactic behavior at low concentrations; as the neutrophil approaches the source of the chemoattractant (eg, invading bacteria), migration is inhibited and the immobilized cell engages bactericidal functions including phagocytosis, degranulation, and the oxidative burst.

\section{Neutrophil Activation, Degranulation}

The capacity of neutrophils to induce tissue damage is undeniable, but this outcome is far from inevitable (Fig 1). The triggering of neutrophil degranulation and respiratory burst is a two-stage process involving an initial prerequisite "priming" step and a second "activation" step. Most secretagogue agonists can only degranulate neutrophils that have been initially primed, for example, via contact with activated endothelium, foreign surfaces, or agents such as lipopolysaccharide. Priming is believed to be an essential "check point" to prevent unregulated neutrophil activation. It is possible that many neutrophils primed in the circulation, for example, in the context of systemic vasculitis, major burns, and pancreatitis, become trapped in the pulmonary microcirculation (see above), and if not induced to migrate by a secondary stimulus ( $e g$, infection) can "de-prime" and be released back into the circulating pool in a quiescent state. While this hypothesis requires experimental testing, it would afford an excellent working model to explain why conditions associated with systemic neutrophil priming cause retention of neutrophils in the lung but often no injury. Priming also inhibits chemotaxis and apoptosis suggesting that this event may serve to "fix" neutrophils at the inflamed site and enhance the functional longevity of these cells. 
Neutrophils possess an array of granules that contain a large number of cytotoxic and immune regulatory molecules. These vesicles are a major source of adhesion molecules and receptors; hence, priming-mediated fusion of secretory granules with the plasma membrane results in a considerable augmentation of adhesive capacity and activation potential. Of note, the four major granule types, namely secretory vesicles (which are endocytic in origin), tertiary, specific, and azurophilic (primary) granules, display differential sensitivity to intracellular $\mathrm{Ca}^{2+}$, which results in a hierarchical pattern of degranulation.22 Granules formed at the later stages of neutrophil maturation (eg, the gelatinase and secretory granules) are also those that are exocytosed most readily, reflecting their differential expression of the fusogenic protein vesicle-associated membrane protein-2.23 This may explain why neutrophils release only a small fraction of their elastase $(2 \%)$ when activated as this protease is stored predominantly within azurophil granules; hence it is likely that neutrophil necrosis contributes far more to elastase release than degranulation. However, elastase has been shown to localize to the leading edge of neutrophils migrating through cultured endothelial cells, 24 but the importance of this phenomenon varies depending on the experimental system used ( $e g$, one recent study 25 found surface neutrophil elastase to be essential for neutrophil transmigration in a cell culture system, whereas a previous report26 found no deficit in neutrophil recruitment to inflamed sites using neutrophil elastase knockout mice).

Neutrophil elastase is an omnivorous enzyme with the capacity to degrade a broad spectrum of structural and immune regulatory proteins including elastin, fibronectin, collagen, $a_{1^{-}}$ antitrypsin, and tissue inhibitors of matrix metalloproteinase, and has been demonstrated to produce emphysema in experimental animals. Elastase is also a potent secretagogue for IL-8 and muc5A and has ciliotoxic and cytotoxic properties. Despite evidence of increased neutrophil numbers and the enhanced elastolytic and adhesive capacity of neutrophils in COPD,27 a direct causal relationship has yet to be established. However, plasma neutrophil elastase levels have been demonstrated to predict the likelihood of ALI,28 and some early studies suggested a possible role for inhibitors of neutrophil elastase in those at risk of ALI following major surgery. Unfortunately, the only large prospective, double-blind, randomized controlled trial29 using the neutrophil elastase inhibitor sivelestat in patients with established ALI (ventilated with low tidal volumes) was halted due to an increase in the 180-day mortality in the treated group, and showed no therapeutic advantage.

A distinctive feature of the neutrophil is its very high intracellular concentration of $\mathrm{Cl}^{-}(80$ to $100 \mathrm{mmol} / \mathrm{L}$ ), which plummets following soluble or particulate stimuli.30 This loss of intracellular $\mathrm{Cl}^{-}$is triggered by Mac-1/CD11b- or Gi-mediated $\mathrm{Cl}^{-}$efflux and modulates both intracellular $\mathrm{Ca}^{2+}$ fluxes and degranulation, suggesting the possibility of using anion channel blockers to control neutrophil degranulation.

A further advance in our understanding of neutrophil secretory mechanisms is the finding that they release neutrophil extracellular traps (NETs) [Fig 2]. Neutrophils have been shown to "spit out" a dense but delicate "web" of primary and secondary granule proteins admixed with chromatin, which serves to trap and kill both bacteria31 and fungi. This event is nicotinamide adenine dinucleotide phosphate (NADPH) oxidase dependent and linked to a distinctive (nonapoptotic, nonnecrotic) form of cell death associated with disintegration of the nuclear envelope and the granule membranes and mixing of NET components.

\section{Neutrophil Activation, Respiratory Burst}

The neutrophil is a professional phagocyte and depends on activation of the multicomponent NADPH oxidase for generating superoxide anions and bacterial killing. Intracellular fungi, such as Candida spp and Staphylococcus aureus, are the organisms classically killed by oxidant-dependent pathways and are also the most problematic pathogens in patients with 
chronic granulomatous disease. The past few years have revealed intriguing new insights into the regulation of the NADPH oxidase, for example the observation that receptormediated activation of PI3K- $\delta$ and $-\gamma$ isoforms results in local PtdIns $(3,4,5) \mathrm{P}_{3}$ generation, 32 which leads to the phosphorylation and recruitment of the oxidase components and assembly of the active NADPH enzyme complex at the site of PI3K activation. Neutrophils derived from mice lacking PI3K- $\gamma 19$ exhibit a profound reduction in the release of reactive oxygen species (ROS) to the environment in response to soluble agonists, while generation of ROS within the phagosome is preserved, demonstrating the feasibility of preventing extracellular oxidant-induced tissue injury without compromising bactericidal capacity.

While it was previously assumed that ROS exert a direct toxic effect on ingested pathogens, previous work suggests that the mechanism may in fact be indirect. The respiratory burst leads to the extrusion of protons into the cytosol, and the resultant ion fluxes produce an environment within the phagosome that is more favorable for the neutral proteases (such as elastase and cathepsins) to digest the contents of the vacuole. 33

\section{Broader Role of the Neutrophil in Innate Immunity}

In the past few years, several articles have pointed to the involvement of the neutrophil in hitherto-unrecognized aspects of the innate immune response. Examples include the capacity of the neutrophil to generate CXCL10/interferon- $\gamma$-inducible protein-10, which acts as an important chemoattractant for activated natural killer and T-helper type 1 cells 34 and for neutrophils to digest and present antigen fragments to dendritic cells, thus serving a critical role in integrating the innate and adaptive limbs of the immune response. Remarkably, it has been proposed that mature postmitotic neutrophils can also "transdifferentiate" into muchlonger-lived cells with macrophage-like characteristics and thereby contribute to resolution and repair at inflamed sites. 35

\section{Resolution of Neutrophilic Inflammation}

Neutrophils migrating to the tissues undergo apoptosis, or programmed cell death, an event that triggers their recognition and phagocytosis by inflammatory macrophages and the subsequent release of antiinflammatory molecules ( $\mathrm{eg}$, IL-10 and transforming growth factor- $\beta 36$ ). The interaction between oxidized phosphatidylserine on the apoptotic cell surface and the class B scavenger receptor CD36 on the macrophage is one mechanism underlying this event.37 For the neutrophil at least, this process is believed to be essential for the "safe" and efficient removal of these otherwise toxic cells and the resolution of the inflammatory response (Fig 3). Exciting new data not only testify to the importance of this event in vivo but also suggest novel therapeutic opportunities to enhance this process through the use of cyclin-dependent kinase inhibitors in models of pulmonary inflammatory disease. 38

Three further recent observations in the field of neutrophil apoptosis/necrosis may have direct relevance to clinical medicine. The first is that neutrophils incubated with noncapsulated clinical isolates of Haemophilus influenzae in vitro are unable to kill these pathogens but instead undergo necrosis with consequent release of histotoxins such as elastase. 39 These data imply a mechanism for $H$ influenzae colonization and airway damage in patients with COPD. Second is the recognition that severe hypoxia ( 0 to $3 \mathrm{kPa}$, as found within an empyema cavity for example) inhibits neutrophil apoptosis and thereby extends the functional life span of these cells rather than inducing cell death (as occurs in the majority of cell types). This may relate to the dominant glycolytic metabolism of neutrophils, which allows them to migrate against an adverse oxygen gradient and retain their phagocytic and antibacterial capacity even in severely oxygen-deplete environments such as abscesses and empyema cavities.40 Finally, it has been reported that cigarette smoke 
impairs the capacity of macrophages to ingest apoptotic neutrophils, 41 perhaps by modification of matrix proteins or cleavage of macrophage scavenger receptors by oxidants.

\section{Therapeutic Implications}

The ideal therapeutic strategy targeting neutrophils would be to abolish their destructive potential without compromising their critical role in host defense, but this has been difficult to achieve. Successful treatments in animal models have frequently proven ineffective or limited by side effects when used in human inflammatory diseases. Improved understanding of neutrophil signal transduction and the precise mechanisms of neutrophil-mediated tissue injury may enable more focused application of targeted antineutrophil therapy. For example, Hartl et al 42 recently described a mechanism whereby neutrophil-derived proteases present in the cystic fibrosis airway cleaved the chemokine receptor CXCR1 from intact neutrophils, disabling their killing capacity; additionally, the cleavage products activated epithelial cells, creating a vicious cycle of infection and inflammation. Inhalation of $a_{1}$-antitrypsin restored airway neutrophil CXCR1 expression and bacterial killing in patients with cystic fibrosis.

While the safety of whole $a_{1}$-antitrypsin has been documented in cystic fibrosis, 43 more protracted studies are required to establish whether clinical benefit will result from this therapy. Likewise, small-molecule inhibitors of the chemokine receptors CXCR1 and CXCR2 are being developed for use in COPD and other inflammatory conditions. Small molecule inhibitors of the PI3K- $\gamma$ are also generating considerable interest as potent antiinflammatory agents. While inhibitors of cyclin-dependent kinases may have multiple effects out with the immune system and may therefore be inappropriate for clinical use, the proof of principle that promoting neutrophil apoptosis can hasten resolution of inflammation may promote the development of other modulators of neutrophil apoptosis as antiinflammatory agents.

The use of macrolide antibiotics is becoming increasingly recognized as a dual antibacterial and antiinflammatory approach, and in many cases the predominant therapeutic effect may be via modulation of neutrophil function; for example, macrolides have been shown to reduce airway neutrophilia in both refractory asthma44 and the bronchiolitis obliterans syndrome, 45 with concomitant clinical improvement. Finally, it is recognized that endogenous mediators such as lipoxins may act as braking signals in inflammation, in part by inhibition of neutrophil PI3K and phospholipase D,46 and the therapeutic potential of structural mimetics of these compounds is currently being explored.

\section{Conclusions}

Our understanding of neutrophil biology, in particular the molecular and cellular mechanisms responsible for their formation and release from the bone marrow, their recruitment, priming and activation within inflamed tissues, and the events resulting in their removal have increased substantially over recent years. The view of the neutrophil as a short-lived, synthetically inert, and rather "monochromatic" cell with limited biodiversity is clearly no longer tenable. The unique transcriptome and proteome of the neutrophil make it a realistic therapeutic target. The challenge will be to retain its peacekeeping and defense functions, while modulating its aggressive tendencies.

\section{Acknowledgments}

The authors' laboratory is funded by the Wellcome Trust, Asthma-UK, MRC, Papworth Hospital, Cambridge NIHR Biomedical Research Centre and the British Lung Foundation. 


\section{Abbreviations}

$\begin{array}{ll}\text { ALI } & \text { acute lung injury } \\ \text { IL } & \text { interleukin } \\ \text { NADPH } & \text { nicotinamide adenine dinucleotide phosphate } \\ \text { NET } & \text { neutrophil extracellular trap } \\ \text { PI3K } & \text { phosphoinositide 3-kinase } \\ \text { ROS } & \text { reactive oxygen species }\end{array}$

\section{References}

1. Quint JK, Wedzicha JA. The neutrophil in chronic obstructive pulmonary disease. J Allergy Clin Immunol. 2007; 119:1065-1071. [PubMed: 17270263]

2. Sparrow D, Glynn RJ, Cohen M, et al. The relationship of the peripheral leukocyte count and cigarette smoking to pulmonary function among adult men. Chest. 1984; 86:383-386. [PubMed: 6467999]

3. Kariyawasam HH, Aizen M, Barkans J, et al. Remodelling and AHR but not cellular inflammation persist after allergen challenge in asthma. Am J Respir Crit Care Med. 2007; 175:896-904. [PubMed: 17272787]

4. Macdowell AL, Peters SP. Neutrophils in asthma. Curr Allergy Asthma Rep. 2007; 7:464-468. [PubMed: 17986378]

5. Hauber HP, Gotfried M, Newman K, et al. Effect of HFA-flunisolide on peripheral lung inflammation in asthma. J Allergy Clin Immunol. 2003; 112:58-63. [PubMed: 12847480]

6. Lee KY, Ho SC, Lin HC, et al. Neutrophil-derived elastase induces TGF- $\beta 1$ secretion in human airway smooth muscle via NF-кB pathway. Am J Respir Cell Mol Biol. 2006; 35:407-414. [PubMed: 16690986]

7. Renshaw SA, Loynes CA, Trushell DM, et al. A transgenic zebrafish model of neutrophilic inflammation. Blood. 2006; 108:3976-3978. [PubMed: 16926288]

8. Sasmono RT, Ehrnsperger A, Cronau SL, et al. Mouse neutrophilic granulocytes express mRNA encoding the macrophage colony-stimulating factor receptor (CSF-1R) as well as many other macrophage-specific transcripts and can transdifferentiate into macrophages in vitro in response to CSF-1. J Leukoc Biol. 2007; 82:111-123. [PubMed: 17438263]

9. Martin C, Burdon PC, Bridger G, et al. Chemokines acting via CXCR2 and CXCR4 control the release of neutrophils from the bone marrow and their return following senescence. Immunity. 2003; 19:583-593. [PubMed: 14563322]

10. Woolhouse IS, Bayley DL, Stockley RA. Sputum chemotactic activity in chronic obstructive pulmonary disease: effect of $a_{1}$-antitrypsin deficiency and the role of leukotriene $\mathrm{B}_{4}$ and interleukin 8. Thorax. 2002; 57:709-714. [PubMed: 12149532]

11. Donnelly SC, Strieter RM, Kunkel SL, et al. Interleukin-8 and development of adult respiratory distress syndrome in at-risk patient groups. Lancet. 1993; 341:643-647. [PubMed: 8095568]

12. Maneechotesuwan K, Essilfie-Quaye S, Kharitonov SA, et al. Loss of control of asthma following inhaled corticosteroid withdrawal is associated with increased sputum interleukin- 8 and neutrophils. Chest. 2007; 132:98-105. [PubMed: 17550933]

13. Doerschuk CM, Beyers N, Coxson HO, et al. Comparison of neutrophil and capillary diameters and their relation to neutrophil sequestration in the lung. J Appl Physiol. 1993; 74:3040-3045. [PubMed: 8366005]

14. Yoshida K, Kondo R, Wang Q, et al. Neutrophil cytoskeletal rearrangements during capillary sequestration in bacterial pneumonia in rats. Am J Respir Crit Care Med. 2006; 174:689-698. [PubMed: 16741152]

15. Ley K, Laudanna C, Cybulsky MI, et al. Getting to the site of inflammation: the leukocyte adhesion cascade updated. Nat Rev Immunol. 2007; 7:678-689. [PubMed: 17717539] 
16. Mizgerd JP, Horwitz BH, Quillen HC, et al. Effects of CD18 deficiency on the emigration of murine neutrophils during pneumonia. J Immunol. 1999; 163:995-999. [PubMed: 10395697]

17. van Buul JD, Allingham MJ, Samson T, et al. RhoG regulates endothelial apical cup assembly downstream from ICAM1 engagement and is involved in leukocyte transendothelial migration. J Cell Biol. 2007; 178:1279-1293. [PubMed: 17875742]

18. Wang S, Voisin MB, Larbi KY, et al. Venular basement membranes contain specific matrix protein low expression regions that act as exit points for emigrating neutrophils. J Exp Med. 2006; 203:1519-1532. [PubMed: 16754715]

19. Hirsch E, Katanaev VL, Garlanda C, et al. Central role for G protein-coupled phosphoinositide 3kinase $\gamma$ in inflammation. Science. 2000; 287:1049-1053. [PubMed: 10669418]

20. Camps M, Rückle T, Ji H, et al. Blockade of PI3K $\gamma$ supresses joint inflammation and damage in mouse models of rheumatoid arthritis. Nat Med. 2005; 11:936-943. [PubMed: 16127437]

21. Maus UA, Backi M, Winter C, et al. Importance of phosphoinositide 3-kinase $\gamma$ in the host defense against pneumococcal infection. Am J Respir Crit Care Med. 2007; 175:958-966. [PubMed: 17322108]

22. Sengeløv H, Follin P, Kjeldsen L, et al. Mobilization of granules and secretory vesicles during in vivo exudation of human neutrophils. J Immunol. 1995; 154:4157-4165. [PubMed: 7535822]

23. Borregaard N, Sørensen OE, Theilgaard-Mönch K. Neutrophil granules: a library of innate immunity proteins. Trends Immunol. 2007; 28:340-345. [PubMed: 17627888]

24. Cepinskas G, Sandig M, Kvietys PR. PAF-induced elastase-dependent neutrophil transendothelial migration is associated with the mobilization of elastase to the neutrophil surface and localization to the migrating front. J Cell Sci. 1999; 112:1937-1945. [PubMed: 10341212]

25. Wang S, Dangerfield JP, Young RE, et al. PECAM-1, a 6 integrins and neutrophil elastase cooperate in mediating neutrophil transmigration. J Cell Sci. 2005; 118:2067-2076. [PubMed: 15840647]

26. Hirche TO, Atkinson JJ, Bahr S, et al. Deficiency in neutrophil elastase does not impair neutrophil recruitment to inflamed sites. Am J Respir Cell Mol Biol. 2004; 30:576-584. [PubMed: 14565940]

27. Keatings VM, Barnes PJ. Granulocyte activation markers in induced sputum: comparison between chronic obstructive pulmonary disease, asthma, and normal subjects. Am J Respir Crit Care Med. 1997; 155:449-453. [PubMed: 9032177]

28. Kodama T, Yukioka H, Kato T, et al. Neutrophil elastase as a predicting factor for development of acute lung injury. Intern Med. 2007; 46:699-704. [PubMed: 17541219]

29. Zeiher BG, Steingrub J, Laterre PF, et al. LY315920NA/S-5920, a selective inhibitor of group IIA secretory phospholipase $\mathrm{A}_{2}$, fails to improve clinical outcome for patients with severe sepsis. Crit Care Med. 2005; 33:1741-1748. [PubMed: 16096451]

30. Busetto S, Trevisan E, Decleva E, et al. Chloride movements in human neutrophils during phagocytosis: characterization and relationship to granule release. J Immunol. 2007; 179:41104124. [PubMed: 17785850]

31. Brinkmann V, Reichard U, Goosmann C, et al. Neutrophil extracellular traps kill bacteria. Science. 2004; 303:1532-1535. [PubMed: 15001782]

32. Condliffe AM, Davidson K, Anderson KE, et al. Sequential activation of class IB and class IA PI3K is important for the primed respiratory burst of human but not murine neutrophils. Blood. 2005; 106:1432-1440. [PubMed: 15878979]

33. Reeves EP, Lu H, Jacobs HL, et al. Killing activity of neutrophils is mediated through activation of proteases by $\mathrm{K}^{+}$flux. Nature. 2002; 416:291-297. [PubMed: 11907569]

34. Tamassia N, Calzetti F, Ear T, et al. Molecular mechanisms underlying the synergistic induction of CXCL10 by LPS and IFN- $\gamma$ in human neutrophils. Eur J Immunol. 2007; 37:2627-2634. [PubMed: 17668902]

35. Araki H, Katayama N, Yamashita Y, et al. Reprogramming of human postmitotic neutrophils into macrophages by growth factors. Blood. 2004; 103:2973-2980. [PubMed: 15070673]

36. Huynh ML, Fadok VA, Henson PM. Phosphatidylserine-dependent ingestion of apoptotic cells promotes TGF- $\beta_{1}$ secretion and the resolution of inflammation. J Clin Invest. 2002; 109:41-50. [PubMed: 11781349] 
37. Greenberg ME, Sun M, Zhang R, et al. Oxidized phosphatidylserine-CD36 interactions play an essential role in macrophage-dependent phagocytosis of apoptotic cells. J Exp Med. 2000; 203:2613-2625. [PubMed: 17101731]

38. Rossi AG, Sawatzky DA, Walker A, et al. Cyclin-dependent kinase inhibitors enhance the resolution of inflammation by promoting inflammatory cell apoptosis. Nat Med. 2006; 12:10561064. [PubMed: 16951685]

39. Naylor EJ, Bakstad D, Biffen M, et al. Haemophilus influenzae induces neutrophil necrosis: a role in chronic obstructive pulmonary disease? Am J Respir Cell Mol Biol. 2007; 37:135-143. [PubMed: 17363778]

40. Walmsley SR, Print C, Farahi N, et al. Hypoxia-induced neutrophil survival is mediated by HIF-1a-dependent NF- $x B$ activity. J Exp Med. 2005; 201:105-115. [PubMed: 15630139]

41. Kirkham PA, Spooner G, Rahman I, et al. Macrophage phagocytosis of apoptotic neutrophils is compromised by matrix proteins modified by cigarette smoke and lipid peroxidation products. Biochem Biophys Res Commun. 2004; 318:32-37. [PubMed: 15110749]

42. Hartl D, Latzin P, Hordijk P, et al. Cleavage of CXCR1 on neutrophils disables bacterial killing in cystic fibrosis lung disease. Nat Med. 2007; 13:1423-1430. [PubMed: 18059279]

43. Martin SL, Downey D, Bilton D, et al. Safety and efficacy of recombinant alpha(1)-antitrypsin therapy in cystic fibrosis. Pediatr Pulmonol. 2006; 41:177-183. [PubMed: 16372352]

44. Simpson JL, Powell H, Boyle MJ, et al. Clarithromycin targets neutrophilic airway inflammation in refractory asthma. Am J Respir Crit Care Med. 2008; 177:148-155. [PubMed: 17947611]

45. Verleden GM, Vanaudenaerde BM, Dupont LJ, et al. Azithromycin reduces airway neutrophilia and interleukin- 8 in patients with bronchiolitis obliterans syndrome. Am J Respir Crit Care Med. 2006; 174:566-570. [PubMed: 16741151]

46. Bonnans C, Fukunaga K, Keledjian R, et al. Regulation of phosphatidylinositol 3-kinase by polyisoprenyl phosphates in neutrophil-mediated tissue injury. J Exp Med. 2006; 203:857-863. [PubMed: 16567384] 


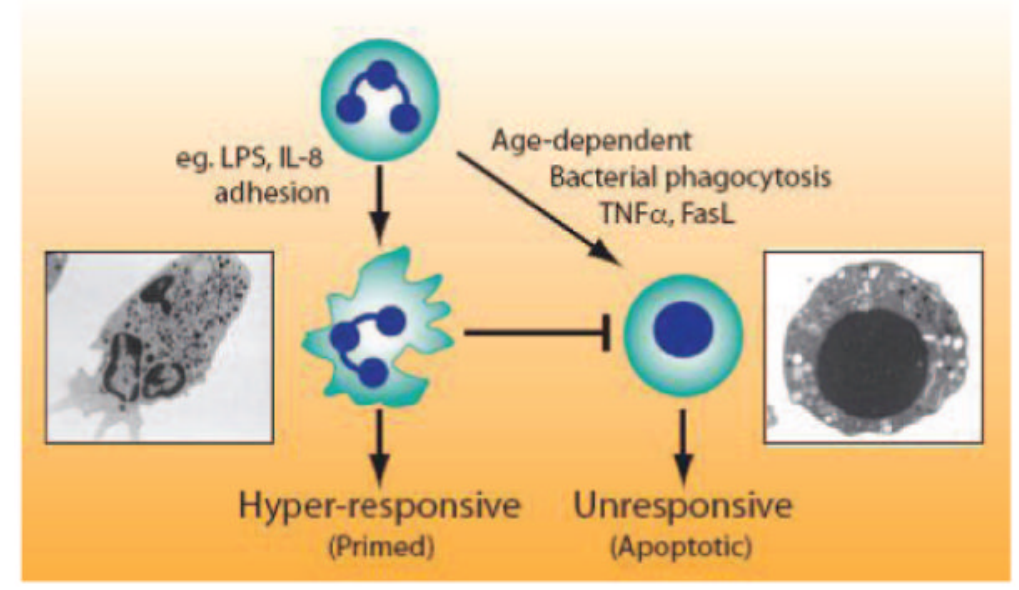

Figure 1.

Neutrophil activation states. When neutrophils adhere to biological surfaces ( $\mathrm{eg}$, fibronectin, collagen) or are exposed to inflammatory mediators such as lipopolysaccharide or IL-8, they become "primed" or hyperresponsive to subsequent activating stimuli, acquiring the capacity to exhibit maximal degranulation and respiratory burst responses and enhanced cytokine and lipid mediator release. Agents that prime neutrophils usually also extend their lifespan by inhibiting apoptosis. The normal age-dependent apoptotic program intrinsic to the neutrophil can be accelerated by ingestion of bacterial pathogens such as Escherichia coli or $S$ aureus (which may promote resolution of bacterial inflammation) or by exposure of these cells to tumor necrosis factor (TNF)a or Fas-ligand (FasL). 


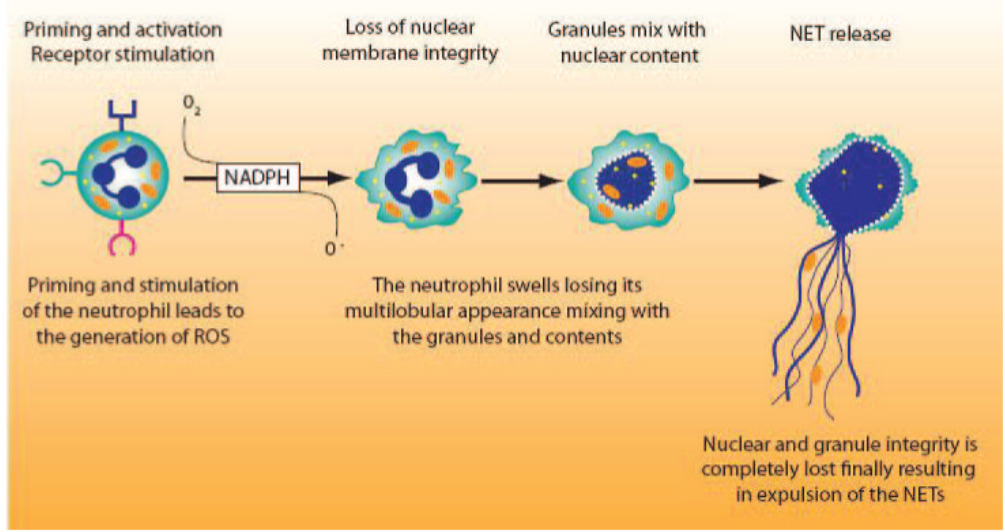

Figure 2.

NET formation. Neutrophils exposed to activating stimuli such as opsonized bacteria become activated and may loose their nuclear integrity, leading to the discharge of granule contents adherent to chromatin strands. These web-like structures may trap and kill bacteria or fungi even after the death of the parent cell. 


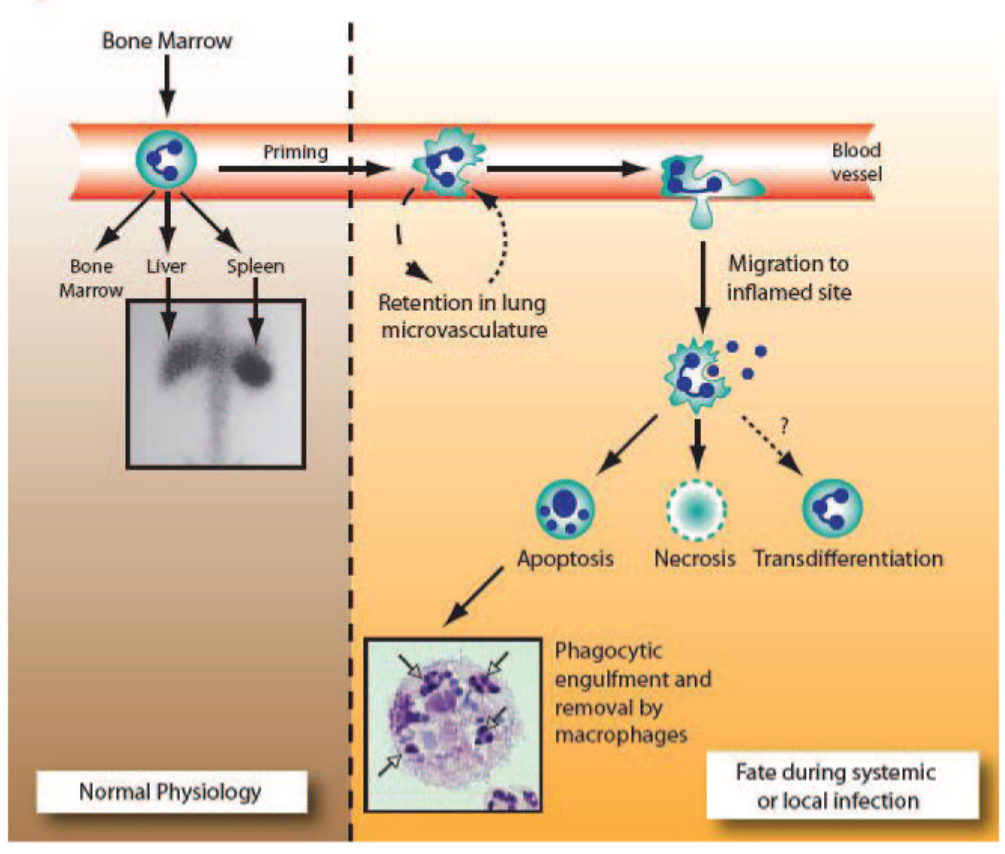

\section{Figure 3.}

Neutrophil fate and function in health and disease. Normal physiology: neutrophils produced in the bone marrow circulate in the bloodstream prior to removal by the bone marrow, or by the liver and spleen (inset is a gamma camera image showing the distribution of ${ }^{111} \mathrm{In}$ tropolone labeled neutrophils in a healthy volunteer at $24 \mathrm{~h}$, depicting the extravascular fate of these cells in the liver, spleen, and bone marrow). Fate during local and systemic inflammation: primed neutrophils undergo rheologic changes leading to retention in the lung microvasculature; such changes are reversible, and in the absence of a further activating stimulus the cells may return to the circulation. If a further activating input is received, the retained neutrophils migrate to the inflamed lung parenchyma to ingest and kill invading bacteria; this may promote apoptosis and the resolution of inflammation, with the apoptotic neutrophils being engulfed by resident macrophages. Conversely, the ingestion of certain bacteria (eg, noncapsulated $H$ influenzae and certain strains of Streptococcus pyogenes) or excessive activation signals may lead to neutrophil necrosis and hence to tissue injury. It has been suggested that neutrophils may undergo transdifferentiation to other cell types (eg, macrophages) and participate in antigen presentation. 